\title{
Dietary diversity and associated factors among adolescents in eastern Uganda: a cross-sectional study
}

\author{
Nathan Isabirye ${ }^{1 *}$, Justine Nnakate Bukenya ${ }^{1 \dagger}$, Mary Nakafeero $^{1 \dagger}$, Tonny Ssekamatte ${ }^{1 \dagger}$, David Guwatudde ${ }^{1 \dagger}$ and \\ Wafaie Fawzi ${ }^{2+}$
}

\begin{abstract}
Background: Globally adolescents constitute over 16\% but in SSA, they make up 23\% of the population. While little is known about diets of these adolescents, rapid changes in physiological and social processes undergone require adequate diets. This study aimed to determine dietary diversity and associated factors among adolescents residing in the lganga -Mayuge HDSS.

Methods: As part of the African Research, Implementation Science, and Education (ARISE) Network, we analysed collected data among 598 adolescents to assess the health status and adolescents' behaviour. Dietary diversity was scored using the 9 food group categories as per the Food and Agriculture Organization -WDDS. Crude and adjusted prevalence rate ratios were estimated using the modified Poisson regression model to identify associated factors.

Results: Among the participants, 45.3\% had a low dietary diversity score. Proportions of adolescents who consumed from the different food categories over a 24-h period were; cereals/roots/tubers (99.7\%), fats \& oils (87.0\%), spices \& beverages (84.1\%), sweets (77.1\%), legumes (66.2\%), other non-vitamin A-rich vegetables $(53.8 \%)$, dark green leafy vegetables (42.3\%), meat/poultry/fish (33.1\%), dairy products (32.9\%), eggs (11.2\%), vitamin A-rich fruits and vegetables (33.4\%) and other fruits (8.2\%). Staying with a single parent or guardian, low socio-economic class, and dependency on home meals was associated with low dietary diversity.
\end{abstract}

Conclusions: Adolescents diets were low in diversity and characterised with low micronutrients source foods, but plenty of fats and oils. Interventions to address contributing factors to the burden ought to target the parenting contexts of the adolescents residing in rural eastern Uganda.

Keywords: Dietary diversity, Parenting, Food intake, Adolescents, Food groupings, Rural households, Micronutrients

\section{Background}

Globally adolescents constitute over 16\% (1.2 billion) of the population, but in Sub Saharan Africa (SSA) they make up 23\% [1]. Adolescents undergo enormous physiological (rapid growth changes during puberty such

\footnotetext{
* Correspondence: isabiryenathan5@gmail.com; isabirye.nathan@yahoo.com ${ }^{1}$ School of Public Health, Makerere University, P.O. Box 7072, Kampala, Uganda

Full list of author information is available at the end of the article
}

as physical activity lean tissue accretion) and social processes (cultural and gender norms; acceptable work types, free time activities, early marriages, and physical activities; changes in access to processed \& unhealthy food markets; food supply deficits at household level) that require an adequate and diverse diet [2] to support the growth spurt and normal pubertal development [3]. Despite the low attention given to adolescents, there is an increase in nutritional demand during this period [4].

C C The Author(s). 2020 Open Access This article is licensed under a Creative Commons Attribution 4.0 International License, which permits use, sharing, adaptation, distribution and reproduction in any medium or format, as long as you give appropriate credit to the original author(s) and the source, provide a link to the Creative Commons licence, and indicate if changes were made. The images or other third party material in this article are included in the article's Creative Commons licence, unless indicated otherwise in a credit line to the material. If material is not included in the article's Creative Commons licence and your intended use is not permitted by statutory regulation or exceeds the permitted use, you will need to obtain permission directly from the copyright holder. To view a copy of this licence, visit http://creativecommons.org/licenses/by/4.0/ The Creative Commons Public Domain Dedication waiver (http://creativecommons.org/publicdomain/zero/1.0/) applies to the data made available in this article, unless otherwise stated in a credit line to the data. 
The majority of nutrition intervention focus on children and mothers and adolescents' demands are not prioritized [2]. Yet, nutrition remains the leading risk factor contributing to the predominant causes of adolescents and adult morbidity and mortality $[2,5]$.

Dietary diversity provides insights into household access to a variety of foods and can be used as a proxy for nutrient adequacy of the diet of individuals [6]. An increase in individual dietary diversity score is related to increased nutrient adequacy of the diet [6]. Eating healthy consists of a balanced and varied diet that constitutes; fresh and natural foods, fruits and vegetables and foods containing vitamins and minerals [7]. It also includes practices of good eating behaviours and habits that contribute to physical and psychological wellbeing [8]. However, diets during adolescence are compromised by low socioeconomic status and food insecurity [9].

While adolescents nutrition is given less priority, 1/3 of the adolescents in the world are obese and more than $10 \%$ in low and middle income countries are underweight [10]. There is a dearth of information on the burden of nutrient adequacy among adolescents in Uganda. A study conducted among adolescent girls indicated a risk of insufficient intake of multiple micronutrients, especially for vitamins A, B12, C, D, E and calcium, and a low intake of essential fatty acids. In addition, stunting and overweight rates stood at $18.6 \%$ and low consumption of animal products such as meat and milk in diet was noted [11]. Paying attention to the nutritional requirements of adolescents is vital in ensuring healthy and productive lives. Adequate nutrition through diverse diets provides an opportunity to correct the deficits suffered during childhood, provide adequate stores of energy for illnesses and pregnancy, and prevents chronic diseases $[8,12,13]$ and adult-onset of nutrition-related diseases and disorders such as osteoporosis and obesity [14]. A balanced nutrient intake provided during this critical period of rapid growth [3] has the potential to reduce morbidity and mortality associated with pregnancy and delivery [15-17], and reduces the risk of delivering low birth-weight babies, consequently preventing an intergenerational cycle of malnutrition $[9,14]$. Adequate nutrition has also shown a positive impact on adolescents' education capacity [18]. It is recommended that adolescents should eat from a variety of foods (low fat animal products, fruits, vegetable, grains, staples), drink plenty of water, and should avoid high consumption of sugars.

While studies conducted in other countries give insights to the burden of malnutrition among adolescents, there is limited evidence on diet diversity and associated factors in Uganda [16-24]. Yet, understanding diet diversity across this age range is vital in the appropriate implementation of targeted nutrition interventions [25].
Makerere University being part of the African Research, Implementation Science, and Education (ARISE) Network, enrolled participants residing in their established surveillance site (Iganga Mayuge Health and Demographic Surveillance Site (IMHDSS) to assess health status and behaviour. Specifically, this analysis describes dietary diversity among adolescents and associated factors in two rural districts of Iganga and Mayuge in Eastern Uganda to inform the designing of interventions that promote adolescents' health.

\section{Methods}

Using a cross-sectional study design, data on dietary diversity was collected during the assessment of health status and behaviour of adolescents residing in Iganga and Mayuge districts. The study utilized the existing IMHDSS data to identify adolescents/households that were enrolled in the survey. Adolescents aged 10-19 by the time of the survey and had resided in IMHDSS for at least one year were considered eligible. A pre-tested questionnaire was used to collect data on sociodemographics, food consumption, and other possible risk factors among 598 adolescents.

\section{Sampling procedure and measurements}

A sample size of 598 adolescents was predetermined based on resources available to conduct the primary study. Adolescents were randomly selected from the IMHDSS database, but stratified in the following two proportions: $80 \%$ from the rural parts, and 20\% from the townships (periurban) of the IMHDSS. Field staffs then used the generated list to visit the households of the adolescents, where they identified the listed adolescents. In case the listed adolescent could not be contacted, traced, or declined to participate in the study, replacement with another adolescent from the nearest household was made. Detailed questionnaires were administered by trained research assistants during a face-to-face interview with participants to obtain socio-demographic data that included: age, parity, education, residence, employment status, socioeconomic status variables, marital status, and past/current use of tobacco, alcohol and other substances. The study adapted and validated the MDDW -questionnaire to contain a list of food groups commonly consumed in eastern Uganda. The lists of food groups were developed using the validated food frequency questionnaire used in the previous surveys to assess effect of standard dose multivitamin supplementation on disease progression in HIVinfected adults conducted in Uganda. This tool was translated into local language, pretested and changes were made to ensure consistency and accuracy during data collection process. The respondents were asked to recall the food and beverages consumed from the time of waking until the respondent went to sleep the past day. This 
helped to indicate on the food list the foods they consumed as appropriate.

\section{Consenting for participation}

A team of consultants made a pre-visit to all IMDHSS before the actual data collection to introduce the study and also to seek permission from local authorities for security reasons. While in the field, informed consent was obtained from all the legal guardians. Upon acceptance of the guardian, assent for adolescents aged less than 18 years was obtained separately. Those above 18 years informed consent was obtained from participants, themselves. Confidentiality was ensured for all study participants by conducting interviews in places with privacy and not using their names for data analysis. We ensured that study participants obtained information about the study, the risks and benefits, and emphasis on the protection of confidentiality. In addition to the informed consent process, an information sheet was given to each respondent. It also included information about key points of contact and phone numbers.

\section{Quality control}

The research assistants were trained before data collection to ensure a mutual understanding of the research protocol as well as the study tools. Research assistants were trained using translated tools to ensure that the survey questions are well understood by both the data collectors and respondents. The study tools were pretested to verify that meaning was not lost during translation as well as to estimate the time required to complete the interview tool.

\section{Data entry and analysis}

Data from the study tools were double entered using Epi Data by independent data clerks to ensure an error-free dataset. STATA SE 14 version was used to analyse the data and to make inferences. Individual Dietary Diversity Score (DDS) was defined as the number of food groups consumed over a $24-\mathrm{h}$ period. The aggregation of foods included in the questionnaire into 9 groups was done according to Food and Agriculture Organisation (FAO) guidelines [26] to create the Women Dietary Diversity Score (WDDS) [27]. Dietary diversity was scored using the 9 food group categories and a cut-off score of $\geq 4$ was considered appropriate (highest sensitivity and specificity) $[28,29]$ hence was used as the minimum cut off point for having a high dietary diversity during analysis. Crude and adjusted prevalence rate ratios (PRR) were estimated using the modified poisson regression model to identify associated factors. The backward stepwise model selection procedure was used to maintain only variables that were significantly associated with the dietary diversity score of $\geq 4$ (outcome of the study) at a $5 \%$ level of significance. The cofounders included in the analysis included; age, sex and socio-economic status of the study participants.

\section{Results}

Data collected from 598 adolescents that participated in the survey were analysed of whom, $52 \%$ were male adolescents and $87.3 \%$ were still in school. As $88.5 \%$ reported both their parents being alive, $70.5 \%$ were living with both parents and $18.1 \%$ had ever engaged in informal employment. Details are illustrated in Table 1.

Table 1 Socio demographic characteristics

\begin{tabular}{|c|c|c|}
\hline Characteristic & $\begin{array}{l}\text { Frequency (n) } \\
N=598\end{array}$ & $\begin{array}{l}\text { Percentage } \\
\%\end{array}$ \\
\hline \multicolumn{3}{|l|}{ Gender } \\
\hline Male & 312 & 52.2 \\
\hline Female & 286 & 47.8 \\
\hline \multicolumn{3}{|l|}{ Age (years) } \\
\hline $10-14$ & 321 & 53.7 \\
\hline $15-19$ & 277 & 46.3 \\
\hline \multicolumn{3}{|l|}{ Highest education level presently } \\
\hline Primary or lower & 419 & 70.1 \\
\hline Secondary or higher & 179 & 29.9 \\
\hline \multicolumn{3}{|l|}{ Living status of parents } \\
\hline Both alive & 527 & 88.5 \\
\hline Single parent or both not alive & 71 & 11.4 \\
\hline \multicolumn{3}{|l|}{ Person adolescent lives with } \\
\hline Both parents & 422 & 70.5 \\
\hline Single parents & 117 & 19.6 \\
\hline Guardian/self & 59 & 9.9 \\
\hline \multicolumn{3}{|c|}{ Gainfully employed in the last 12 months } \\
\hline No & 490 & 81.9 \\
\hline Yes & 108 & 18.1 \\
\hline \multicolumn{3}{|l|}{ Social Economical Status (SES) } \\
\hline Poorest & 184 & 30.8 \\
\hline Poor & 104 & 17.4 \\
\hline Middle class & 103 & 17.2 \\
\hline Rich & 90 & 15.0 \\
\hline Richest & 117 & 19.6 \\
\hline \multicolumn{3}{|l|}{ Eat from restaurant } \\
\hline Never & 510 & 85.3 \\
\hline At least once a week & 88 & 14.7 \\
\hline \multicolumn{3}{|l|}{ Household size } \\
\hline Up to 5 people & 116 & 19.4 \\
\hline 6-10 people & 391 & 65.4 \\
\hline More than 10 & 91 & 15.2 \\
\hline
\end{tabular}


Low dietary diversity among participants stood at $45.32 \%$. Regarding consumption of an item from the food groups in the previous $24 \mathrm{~h}$, the majority (99.7\%) of adolescents reported having consumed cereals/roots/tubers, $(87.0 \%)$ fats \& oils, (84.1\%) spices \& beverages, and 77.1\% consumed sweets. Among the study participants, pulses, nuts, seeds (legumes), and other vegetables (nonvitamin A rich), were averagely consumed ranging from 50 to $66.2 \%$. The least consumed foods included; dark green leafy vegetables (42.3\%), meat/poultry/fish (33.1\%), dairy products $(32.9 \%)$, eggs (11.2\%), vitamin A-rich fruits and vegetables (33.4\%) and other fruits (8.2\%). Details are illustrated in Table 2 .

In the multivariable model, adolescents staying with a single parent and those staying with a guardian or by themselves were more likely to consume a less diverse diet compared to those staying with both parents $($ aPRRs $=1.31,1.39$ Vs aPRR 1.0; 95\%CI $)$. Adolescents living in households of the lowest socio-economic class were more likely to have a low dietary diversity score compared to those in higher social-economic classes (aPRRs 0.58,0.74,0.58 Vs aPRR 1.0; 95\%CI). Adolescents who ate from a restaurant at least once a week were $40 \%$ more likely to have a high dietary diversity score compared to those who depended on household meals. Age, education, employment status, and household size were non-significant factors in determining dietary diversity among these adolescents. Details are illustrated in Tables 3 \& 4 .

\section{Discussion}

Based on the findings, a considerable proportion of adolescents residing in the IMHDSS had a low dietary diversity. The majority of the adolescents reported consuming high proportions of fats and oils, cereals and low intakes of micronutrient sources (vegetables and fruits). The contributing factors to low diversity among the study participants included; staying with a single parent or guardian, low socio-economic status, and dependency on household meals. Age, gender, education level, employment status, and household size were nonsignificant. This region being majorly rural, low socioeconomic status may be of influence to adolescents' low diet diversity.

Similar findings were reported by Ochola et al. in a systematic review which observed limited dietary diversity mainly comprising of plant-based food source, with limited animal foods, fruits and vegetable intakes among adolescents residing in rural communities $[18,19]$. The majority $(83 \%)$ of the IMHDSS is rural and the residents in the area are mostly subsistence farmers, whose diets are predominantly plant-based $[30,31]$. The prevalence of low diet diversity has an implication on nutritional status and is a pointer to the reducing quality of diets in the IMHDSS. The findings may be explained by the poor farming methods which focus on a few crops in the Mayuge and Iganga districts [30].

A significant proportion of adolescents reported high consumption of fats/oils and beverages; with low intakes of animal source foods, micronutrient source fruits and vegetables. Similar findings were observed by Adam et al., which showed a marked positive relationship between low-income population and increased intake of fats and sweetened beverages. Majority of residents being rural, we still observe a shift in diet and this may be partly explained by the increase in the availability of

Table 2 Adolescents intakes presented by food groups and dietary diversity

\begin{tabular}{|c|c|c|}
\hline Food type & $\begin{array}{l}\text { Consumed } \\
N=598 \\
\text { (n) }\end{array}$ & $\begin{array}{l}\text { Percentage that } \\
\text { Consumed } \\
(\%)\end{array}$ \\
\hline Grain, white roots and tuber, $\&$ plantains & 596 & 99.7 \\
\hline Pulses, nuts and seeds & 396 & 66.2 \\
\hline Diary & 197 & 32.9 \\
\hline Meat, poultry and fish & 198 & 33.1 \\
\hline Eggs & 67 & 11.2 \\
\hline Dark green leafy vegetable & 253 & 42.3 \\
\hline Vitamin A-rich fruits and vegetables & 200 & 33.4 \\
\hline Other vegetables & 322 & 53.8 \\
\hline Other fruit & 49 & 8.2 \\
\hline Oils and fats & 520 & 87.0 \\
\hline Sweets & 461 & 77.1 \\
\hline Spices and beverages & 503 & 84.1 \\
\hline Low dietary diversity (LDD) $(<4$ food groups) & 271 & 45.3 \\
\hline
\end{tabular}

Note: Dietary diversity was scored using the 9 food group categories and a cut off of $<4$ was considered as LDD 
Table 3 Dietary diversity among adolescents in the IMHDSS

\begin{tabular}{|c|c|c|c|c|}
\hline \multirow[t]{2}{*}{ Characteristic } & \multirow[t]{2}{*}{$N=598$} & \multicolumn{2}{|c|}{ dietary diversity range $\mathrm{n}$ (row \%) } & \multirow[b]{2}{*}{$p$-value } \\
\hline & & $<4$ & $\geq 4$ & \\
\hline \multicolumn{5}{|l|}{ Gender } \\
\hline Male & 312 & $144(53.1)$ & $168(51.4)$ & \multirow[t]{2}{*}{0.668} \\
\hline Female & 286 & $127(46.9)$ & $159(48.6)$ & \\
\hline \multicolumn{5}{|l|}{ Age (years) } \\
\hline $10-14$ & 321 & $156(57.6)$ & $165(50.5)$ & \multirow[t]{2}{*}{0.083} \\
\hline $15-19$ & 277 & $115(42.4)$ & $162(49.5)$ & \\
\hline \multicolumn{5}{|c|}{ Highest education level attained so far } \\
\hline Primary (1-7 years) & 419 & $194(71.6)$ & $225(68.8)$ & \multirow[t]{2}{*}{0.46} \\
\hline Secondary (> 7 years) & 179 & $77(28.4)$ & $102(31.2)$ & \\
\hline \multicolumn{5}{|c|}{ Person adolescent lives with } \\
\hline Both parents & 422 & $173(63.8)$ & $249(76.1)$ & \multirow[t]{3}{*}{$0.004^{*}$} \\
\hline Single parents & 117 & $64(23.6)$ & $53(16.2)$ & \\
\hline Guardian & 59 & $34(12.5)$ & $25(7.6)$ & \\
\hline \multicolumn{5}{|c|}{ Gainfully employed in the last 12 months } \\
\hline No & 490 & $221(81.5)$ & $269(82.3)$ & \multirow[t]{2}{*}{0.821} \\
\hline Yes & 108 & $50(18.5)$ & $58(17.7)$ & \\
\hline \multicolumn{5}{|l|}{ Socio- economic status } \\
\hline Poorest & 184 & $114(42.1)$ & $70(21.4)$ & \multirow[t]{5}{*}{$<0.001^{*}$} \\
\hline Poor & 104 & $42(15.5)$ & $62(19)$ & \\
\hline Middle & 103 & $36(13.3)$ & $67(20.5)$ & \\
\hline Rich & 90 & $39(14.4)$ & $51(15.6)$ & \\
\hline Richest & 117 & $40(14.8)$ & $77(23.5)$ & \\
\hline \multicolumn{5}{|l|}{ Eat from restaurant } \\
\hline Never & 510 & $244(90.0)$ & $266(81.3)$ & \multirow[t]{2}{*}{$0.003^{*}$} \\
\hline Atleast once a week & 88 & $27(10.0)$ & $61(18.7)$ & \\
\hline \multicolumn{5}{|l|}{ Household size } \\
\hline Up to 5 people & 116 & $58(21.4)$ & $58(17.7)$ & \multirow[t]{3}{*}{0.485} \\
\hline 6-10 people & 391 & $171(63.1)$ & $220(67.3)$ & \\
\hline More than 10 & 91 & $42(15.5)$ & $49(15.0)$ & \\
\hline
\end{tabular}

*5\% level of significance

Note: 9 food categories were considered as recommended by FAO. Individual dietary diversity is categorized as "low dds" if less than 4 food groups consumed, and as "diverse" if $\geq 4$

cheap oils and fats on low-income state markets [32, 33]. Moreover, in Uganda, oils and sweeteners are packed in small quantities increasing availability and affordability.

The study revealed the existence of factors that affect adolescents differently and often relate to the unequal distribution of dietary intakes as observed in other studies $[18,19]$. Single parenting in households where these adolescents reside was associated with a low dietary diversity score. Similar findings have been observed in studies done on the relationship between parenting and dietary intake among children [34-36]. Adolescents need extra support to avoid low- quality diets, yet single parents in a resource-poor setting are usually socially and economically constrained [37]. This involves persistent strains such as economic hardship, loneliness, single parenting hardships, reduced on-farm workforce and may explain the negative impact on dietary diversity among adolescents staying with single parents or those who lost one or both parents.

Another important finding was the positive association of low dietary diversity and being a member of a low socio-economic class household. This is similar to findings from several studies previously conducted in LMICs, which reported low socio-economic status as being associated to poor diets and nutritional status of adolescents [38-40]. What may contribute to low dietary 
Table 4 Factors associated with dietary intake among adolescents in IMHDSS

\begin{tabular}{|c|c|c|c|c|}
\hline \multirow{3}{*}{$\overline{\text { Gender }}$} & \multirow[b]{2}{*}{ N (\%) } & \multirow{2}{*}{$\begin{array}{l}\text { Number with } \\
\text { Low dietary diversity } n(\%)\end{array}$} & \multirow{2}{*}{$\begin{array}{l}\text { Unadjusted } \\
\text { PRR (95\% Cl) }\end{array}$} & \multirow{2}{*}{$\begin{array}{l}\text { Adjusted } \\
\text { PRR }(95 \% \mathrm{Cl}) \\
\end{array}$} \\
\hline & & & & \\
\hline & \multicolumn{4}{|c|}{ Gender } \\
\hline Male & 312 & $168(51.4)$ & 1.00 & 1.00 \\
\hline Female & 286 & $159(48.6)$ & $0.96(0.81-1.15)$ & $0.96(0.79-1.14)$ \\
\hline \multicolumn{5}{|l|}{ Age (Years) } \\
\hline $10-14$ & 321 & $165(50.5)$ & 1.00 & 1.00 \\
\hline $15-19$ & 277 & $162(49.5)$ & $0.85(0.71-1.02)$ & $0.96(0.79-1.15)$ \\
\hline \multicolumn{5}{|c|}{ Highest education level attained so far } \\
\hline Primary ( $1-7$ years) & 419 & $225(68.8)$ & 1.00 & 1.00 \\
\hline Secondary ( $>7$ years) & 179 & $102(31.2)$ & $0.93(0.76-1.13)$ & $1.10(0.87-1.39)$ \\
\hline \multicolumn{5}{|c|}{ Person adolescent lives with } \\
\hline Both parents & 422 & $249(76.1)$ & 1.00 & 1.00 \\
\hline Single parents & 117 & $53(16.2)$ & $1.33(1.09-1.63)$ & $1.31(1.08-1.59)^{*}$ \\
\hline Guardian & 59 & $25(7.6)$ & $1.41(1.1-1.8)$ & $1.39(1.09-1.76)^{*}$ \\
\hline \multicolumn{5}{|c|}{ Gainfully employed in the last 12 months } \\
\hline No & 490 & $269(82.3)$ & 1.00 & 1.00 \\
\hline Yes & 108 & $58(17.7)$ & $1.03(0.82-1.29)$ & $1.09(0.85-1.39)$ \\
\hline \multicolumn{5}{|c|}{ Socio-economic status (SES) } \\
\hline Poorest & 184 & $70(21.4)$ & 1.00 & 1.00 \\
\hline Poor & 104 & $62(19)$ & $0.65(0.5-0.85)$ & $0.66(0.51-0.85)^{*}$ \\
\hline Middle & 103 & $67(20.5)$ & $0.56(0.42-0.75)$ & $0.58(0.43-0.77)^{*}$ \\
\hline Rich & 90 & $51(15.6)$ & $0.7(0.54-0.91)$ & $0.74(0.57-0.97)^{*}$ \\
\hline Richest & 117 & $77(23.5)$ & $0.55(0.42-0.73)$ & $0.58(0.45-0.77)^{*}$ \\
\hline \multicolumn{5}{|l|}{ Eat from restaurant } \\
\hline Never & 510 & $266(81.3)$ & 1.00 & 1.00 \\
\hline Atleast once a week & 88 & $61(18.7)$ & $0.64(0.46-0.89)$ & $0.59(0.44-0.83)^{*}$ \\
\hline \multicolumn{5}{|l|}{ Household size } \\
\hline Up to 5 people & 116 & $58(17.7)$ & 1.00 & 1.00 \\
\hline 6-10 people & 391 & $220(67.3)$ & $0.87(0.71-1.08)$ & $1.04(0.84-1.28)$ \\
\hline More than 10 & 91 & 49 (15.0) & $0.92(0.69-1.23)$ & $1.21(0.89-1.62)$ \\
\hline
\end{tabular}

*5\% level of significance

Note: Sex, age and SES maintained as potential universal confounders

diversity in these households is the dependency on mainly home-grown staples and legumes with limitation in access to other important healthy foods which are available in the local markets.

The study revealed a positive association between eating away from home in a restaurant and dietary diversity. This is in contrast to the previous studies that indicated low diverse diets among adolescents who consumed foods away from home [41, 42]. This variation may be explained by the difference in the food types and mode of preparations between fast-foods and the local restaurants found in the IMHDSS. It turns out an opportunity to consume a variety of foods from restaurants which prepare a variety of foods compared to the home meals which are constrained to only foods grown in the home backyard.

\section{Study limitation}

The self-report using a food list used to collect data can be biased for true dietary intakes and may not reflect the routine diet pattern of participants. However, the use of the food lists is a good technique of estimating diversity in diets of individuals other than macro/micronutrient intake estimates for detailed analysis in diets of individuals. Having a detailed estimate of food intake useful in assessing nutrient intakes contained in the diets of individuals. Besides, research assistants were also trained before data collection to ensure consistency and accuracy 
during the entire data collection process. They were further trained on using illustrations/aiding objects to assist participants in reporting of food types consumed by an individual. At each end of the day, supervisors were assigned to review tools for accuracy and completeness of data collection and this helped in the correction of errors.

\section{Conclusion}

Adolescents residing in rural eastern Uganda are at risk of having low dietary diversity. Their diets are characterized by high consumption of fats and oils but with low intake of micronutrient-rich foods such as fruits and vegetables. This type of diet is nutritionally inadequate, and may perpetuate the double burden of malnutrition among adolescents. Nutrition-related intervention, ought to target the parenting contexts of adolescents. Further studies to understand the quantities and nutrients contained in the diets of these adolescents are recommended.

\section{Abbreviations}

IMHDSS: Iganga Mayuge health and demographic surveillance site WDDS: Women's dietary diversity score; DDS: Dietary diversity score; PRR: Prevalence rate ratios; FAO: Food and agriculture organization; LMICs: Low and middle-income countries; ARISE: Africa research, implementation science, and education network; SES: Socio-economic status

\section{Acknowledgements}

We acknowledge the contribution of field supervisors and data collectors, the Iganga-Mayuge District Health \& Demographic Surveillance Site technical staffs, as well as the contribution of the study participants.

\section{Authors' contributions}

$\mathrm{NI}$, JNB, MN, TS, DG, FW; conceived and developed the study protocol. NI, JNB, MN, DG, FW; conducted the study. MN, DG, FW, Nl; analysed and interpreted the participants' data. NI, JNB, TS, DG \&FW; contributed to the writing of the manuscript. All authors read and approved the final manuscript.

\section{Funding}

Funding for the ARISE Adolescent Health Study was provided by the Department of Global Health and Population at Harvard T.H. Chan School of Public Health." The funder had no role in the design of the study and collection, analysis, and interpretation of data and in writing the manuscript.

\section{Availability of data and materials}

The datasets used and/or analysed during the current study are available from the corresponding author on reasonable request.

\section{Ethics approval and consent to participate}

Ethical approval was sought from Makerere University School of Public Health Higher Degrees and Research Ethics Committee and Uganda National Council for Science and Technology (protocol number: SS 3996). Written informed consent was obtained from adolescents above 18 years and all the legal guardians for those less than 18 years, from whom assent was obtained.

\section{Consent for publication}

Not applicable.

\section{Competing interests}

The authors declare no competing interests.

\section{Author details}

${ }^{1}$ School of Public Health, Makerere University, P.O. Box 7072, Kampala, Uganda. 'Department of Global Health and Population, Harvard University, Boston, USA.

Received: 11 June 2019 Accepted: 6 April 2020

Published online: 19 April 2020

\section{References}

1. UNICEF. UNICEF Data: Monitoring the Situation of Children and Women. 2018 Feb 2018 31-May-2018]; Available from: https://data.unicef.org/topic/ adolescents/demographics/\#

2. Christian P, Smith ER. Adolescent Undernutrition: global burden, physiology, and nutritional risks. Ann Nutr Metab. 2018;72(4):316-28.

3. Soliman A, De Sanctis V, Elalaily R. Nutrition and pubertal development. Indian J Endocrinol Metab. 2014;18(Suppl 1):S39.

4. Nabugoomu, J., Adolescent maternal nutrition and health in Uganda: voices from the community. 2018

5. Black RE, et al. Maternal and child undernutrition and overweight in lowincome and middle-income countries. Lancet. 2013:382(9890):427-51.

6. Kennedy G, Ballard T, Dop MC. Guidelines for measuring household and individual dietary diversity: Food and Agriculture Organization of the United Nations. 2011. Available from: http://www.fao.org/3/a-i1983e.pdf.

7. Povey $\mathrm{R}$, et al. Interpretations of healthy and unhealthy eating, and implications for dietary change. Health Educ Res. 1998;13(2):171-83.

8. Polivy J, Herman CP. Mental health and eating behaviours: a bi-directional relation. Can J Public Health/Rev Can de Sante'e Publique. 2005;6(3):S43-6.

9. Wassie MM, et al. Predictors of nutritional status of Ethiopian adolescent girls: a community based cross sectional study. BMC Nutr. 2015;1(1):20.

10. Jai KD, et al. Nutrition in adolescents: physiology, metabolism, and nutritional needs. Ann N Y Acad Sci. 2017:1393(1):21-33.

11. Berg T, Magala-Nyago C, Iversen PO. Nutritional status among adolescent girls in children's homes: anthropometry and dietary patterns. Clin Nutr. 2017;37(3):926-33

12. Niva M. 'All foods affect health': understandings of functional foods and healthy eating among health-oriented Finns. Appetite. 2007;48(3):384-93.

13. Hoelscher DM, et al. Designing effective nutrition interventions for adolescents. J Acad Nutr Diet. 2002;102(3):S52-63.

14. WHO, Adolescent nutrition: a review of the situation in selected South-East Asian countries. 2006

15. Thurnham DI. Nutrition of adolescent girls in low-and middle-income countries. Sight Life. 2013:27:26-37.

16. Belachew T, et al. Predictors of chronic food insecurity among adolescents in Southwest Ethiopia: a longitudinal study. BMC Public Health. 2012:12(1):604.

17. Belachew T, et al. Food insecurity and linear growth of adolescents in Jimma zone, Southwest Ethiopia. Nutr J. 2013:12(1):55.

18. Ochola S, Masibo PK. Dietary intake of schoolchildren and adolescents in developing countries. Ann Nutr Metab. 2014;64(Suppl. 2):24-40.

19. Pradeilles, R., Neighbourhood and household socio-economic influences on diet and anthropometric status in urban South African adolescents. 2015, (c) Rebecca Pradeilles.

20. Crush J, Frayne B. Supermarket expansion and the informal food economy in southern African cities: implications for urban food security. J South Afr Stud. 2011;37(4):781-807.

21. Feighery, J., et al., Intersections of youth and food security. Report submitted to the United States Agency for International Development. URL: http://pdf. usaid. gov/pdf docs/PNADU952.pdf (Accessed 12 May 2015), 2011.

22. Hadley $C$, et al. Rapidly rising food prices and the experience of food insecurity in urban Ethiopia: impacts on health and well-being. Soc Sci Med. 2012;75(12):2412-9.

23. Aurino E, Fernandes M, Penny ME. The nutrition transition and adolescents' diets in low-and middle-income countries: a cross-cohort comparison. Public Health Nutr. 2017;20(1):72-81.

24. Steyn NP, Labadarios D, Nel JH. Factors which influence the consumption of street foods and fast foods in South Africa-a national survey. Nutr J. 2011; 10(1):104

25. Winpenny EM, et al. Change in diet in the period from adolescence to early adulthood: a systematic scoping review of longitudinal studies. Int J Behav Nutr Phys Activ. 2017;14(1):60.

26. 360, F.a.F. Minimum Dietary Diversity for Women: A Guide for Measurement. Rome: FAO. 2016. 
27. FAO, Guidelines for measuring household and individual dietary diversity. 2010.

28. Bullecer ER, et al. Dietary diversity score as an Indicator of nutritional adequacy of diets among 16-19-year-old adolescents. Acta Med Philipp. 2012:46(1):27-33.

29. Habte T, Krawinkel M. Dietary diversity score: a measure of nutritional adequacy or an Indicator of healthy diet. J Nutr Health Sci. 2016;3(3):303.

30. governement, M.d.l., District development plan II 2015/16-2019/20. 2015.

31. UBOS. The National Population and housing census 2014 - area specific profile series. Uganda: Kampala; 2017.

32. Popkin BM. Global nutrition dynamics: the world is shifting rapidly toward a diet linked with noncommunicable diseases. Am J Clin Nutr. 2006;84(2):289-98,

33. Popkin BM, Adair LS, Ng SW. Global nutrition transition and the pandemic of obesity in developing countries. Nutr Rev. 2012;70(1):3-21.

34. Larsen JK, et al. How parental dietary behaviour and food parenting practices affect children's dietary behaviour. Interacting sources of influence? Appetite. 2015;89:246-57.

35. Williams, D., Parents shape whether their children learn to eat fruits and vegetables. 2008.

36. Russell SJ, Hughes K, Bellis MA. Impact of childhood experience and adult well-being on eating preferences and behaviours. BMJ Open. 2016;6(1): e007770.

37. Amato PR. The consequences of divorce for adults and children. J Marriage Fam. 2000;62(4):1269-87.

38. Labadarios D, Steyn NP, Nel J. How diverse is the diet of adult south Africans? Nutr J. 2011;10(1):33.

39. Maziya, N., Adolescent nutritional status and its association with village-level factors in Tanzania. 2014

40. Morseth MS, et al. Dietary diversity is related to socioeconomic status among adult Saharawi refugees living in Algeria. BMC Public Health. 2017; 17(1):621

41. Lachat $\mathrm{C}$, et al. Eating out of home and its association with dietary intake: a systematic review of the evidence. Obes Rev. 2012;13(4):329-46.

42. Nago ES, et al. Association of out-of-home eating with anthropometric changes: a systematic review of prospective studies. Crit Rev Food Sci Nutr. 2014:54(9):1103-16.

\section{Publisher's Note}

Springer Nature remains neutral with regard to jurisdictional claims in published maps and institutional affiliations.

Ready to submit your research? Choose BMC and benefit from:

- fast, convenient online submission

- thorough peer review by experienced researchers in your field

- rapid publication on acceptance

- support for research data, including large and complex data types

- gold Open Access which fosters wider collaboration and increased citations

- maximum visibility for your research: over $100 \mathrm{M}$ website views per year

At $\mathrm{BMC}$, research is always in progress.

Learn more biomedcentral.com/submissions 